\title{
EMPATHY THEORY AND THE LAPLACE TRANSFORM
}

\author{
NIKO SAUER \\ Faculty of Science, University of Pretoria \\ Pretoria 0002, South Africa \\ E-mail: nikos@friedrichs.up.ac.za
}

\begin{abstract}
This paper is concerned with double families of evolution operators employed in the study of dynamical systems in which cause and effect are represented in different Banach spaces. The main tool is the Laplace transform of vector-valued functions. It is used to define the generator of the double family which is a pair of unbounded linear operators and relates to implicit evolution equations in a direct manner. The characterization of generators for a special class of evolutions is presented.
\end{abstract}

1. Introduction. The Cauchy problem

$$
\begin{aligned}
\frac{d u}{d t} & =A u(t), \\
\left.u\right|_{t=0} & =y,
\end{aligned}
$$

where $A$ is a closed linear operator with domain $\mathfrak{D}(A)$ and range $\mathfrak{R}(A)$ both contained in a Banach space $Y$ is effectively studied by finding a semigroup $E(t): Y \rightarrow Y$ of bounded linear operators with $A$ its infinitesimal generator. Characterizations of the generators of semigroups in terms of properties of their resolvents are well-known (see e.g. [HP57]).

In this paper we study implicit Cauchy problems of the form

$$
\begin{aligned}
\frac{d}{d t}[B u(t)] & =A u(t), \\
\lim _{t \rightarrow 0+} B u(t) & =y \in Y,
\end{aligned}
$$

where $A$ and $B$ are unbounded linear operators with a common domain $\mathfrak{D}$ contained in a Banach space $X$ and $\mathfrak{R}(A)$ as well as $\mathfrak{R}(B)$ in a Banach space $Y$.

In many applications the operator $B$ is not closable so that there is no way in which the "time derivative" or the limit at $t=0+$ can commute with $B$. For that reason it seems to be appropriate to study (1) as it stands. Our approach to the problem is intuitively

1991 Mathematics Subject Classification: Primary 47D06; Secondary 34G10.

Research supported by the South African Foundation for Research Development.

The paper is in final form and no version of it will be published elsewhere. 
to represent the solution of (1) in the form $u(t)=S(t) y$ with the "solution operator" $S(t)$ defined on the space $Y$ and range in $X$. At the same time we consider $v(t)=B S(t)$ to be emanating from $y \in Y$ and represented as $v(t)=E(t) y$ with the operator $E(t)$ defined on $Y$ and range in $Y$. The evolution of the system is decribed by thinking of each $v(t)$ as an initial state for $u$ at later times. This notion is described symbolically by $u(t+s)=S(s) v(t)$, which lies at the core of empathy theory. The paper is concerned with the development of a theory of a "double family" $\langle S(t), E(t)\rangle$ of evolution operators which is consistent with problems of the form (1). This approach was introduced in a restricted way in [SS89] and studied for a special case in [CS94]. A brief outline of the theory is also given in [S95].

In the study of semigroups the notion of infinitesimal operator/generator and the observation that, under certain conditions, the Laplace transform of the semigroup is the resolvent of the infinitesimal generator is crucial. In the abstract study of (1) the notion of infinitesimal generator is bound to involve the operator $B$ and gives rise to difficulties with the kernel of $B$ [Sau82, SS87]. Thus it is expedient to develop the theory entirely with the aid of the Laplace transform which will be an important tool in this study.

It should be noted that implicit Cauchy problems such as (1) are treated in [CaSh76], but from a different perspective. In [Fav79] the problem is studied with the aid of the Laplace transform under the assumption that the operator $A$ is injective. In that paper the notion of integrated semigroup, introduced formally in [Are87], appears implicitly. In [AF93] the theory of integrated semigroups is employed to study special implicit equations of the form (1). For the case $B=I$, the identity, integrated semigroups may be viewed as special cases of regularized semigroups [daP66] or C-semigroups [DP87] which, under restricted circumstances, may be viewed as a special case of the theory presented here [CS94]. A comprehensive survey of C-semigroups and integrated semigroups is presented in [DeL94]. It should be noted that the notion of empathy is related to the notion of existence and uniqueness family employed in [DeL91] (see also [DeL94, Chap. XVI).

In Section 2 the notion of empathy is defined and the properties of the operators $E(t)$ and $S(t)$ are obtained under general conditions. This investigation is continued in Section 3 where additional results are obtained when boundedness conditions are imposed on the resolvents. In Section 4 we use the Post-Widder inversion theorem to obtain additional information on the structure of empathies. In particular, it is shown that the operators $E(t)$ map $Y$ into a fixed closed linear subspace $Y_{E}$ which plays a central role in the theory. In Section 5 we define the notion of generator of an empathy which is an operator pair $\langle A, B\rangle$ relating directly to the Cauchy problem (1). In Section 6 we obtain a uniqueness result for the representation $u(t)=S(t) y$ of solutions of (1). The remaining sections are devoted to the characterization of the generators of uniformly bounded empathies. It turns out that the characterization is in general only possible if the space $Y$ has the Radon-Nikodým property (see [DU77] for an extensive treatment of this property).

2. Empathy theory. Let $X$ and $Y$ be Banach spaces and let $\mathcal{E}=\{E(t): Y \rightarrow Y \mid$ $t>0\}$ and $\mathcal{S}=\{S(t): Y \rightarrow X \mid t>0\}$ be two families of bounded linear operators. We shall assume that for every $y \in Y$ and $\lambda>0$ the Laplace transforms 


$$
R(\lambda) y=\int_{0}^{\infty} e^{-\lambda t} E(t) y d t, \quad P(\lambda) y=\int_{0}^{\infty} e^{-\lambda t} S(t) y d t
$$

exist as Lebesgue integrals in the sense that $e^{-\lambda \cdot} E(\cdot) y \in L^{1}(0, \infty ; Y)$ and $e^{-\lambda} \cdot S(\cdot) y \in$ $L^{1}(0, \infty ; X)$. The double family $\langle\mathcal{S}, \mathcal{E}\rangle$ is called an empathy if the following conditions hold:

$$
\begin{aligned}
& S(t+s)=S(t) E(s) \quad \text { for arbitrary } s, t>0, \\
& \text { For some } \xi>0, P(\xi) \text { is invertible. }
\end{aligned}
$$

Note that the behaviour of $S(t) y$ and $E(t) y$ at $t=0$ is not specified and that no boundedness conditions are imposed on the linear operators $R(\lambda)$ and $P(\lambda)$. We shall refer to (2) as the empathy relation.

Proposition 2.1. If $\langle\mathcal{S}, \mathcal{E}\rangle$ is an empathy then $\mathcal{E}$ is a semigroup.

Proof. From (2) it is clear that $S(t+r+s) y=S(t) E(r+s) y=S(t) E(r) E(s) y$ for positive $r, s$ and $t$. Taking the Laplace transform with respect to $t$ yields $P(\xi)[E(r+$ $s) y-E(r) E(s) y]=0$ and the result follows from (3).

ThEOREM 2.2. The norms $\|E(t)\|$ and $\|S(t)\|$ are locally uniformly bounded in $(0, \infty)$ and the families $\mathcal{E}$ and $\mathcal{S}$ are strongly continuous on $(0, \infty)$.

Pr o of. Since $\mathcal{E}$ is a semigroup and $t \rightarrow E(t) y$ is measurable, the statement regarding $E(t)$ is well-known [Miy51; HP57]. The strong continuity of $\mathcal{S}$ now follows from the empathy relation and the boundedness of $S(t)$. The local boundedness of $\|S(t)\|$ follows from the uniform boundedness principle.

We collect a number of important identities in the following result.

Lemma 2.3. For arbitrary positive $\lambda, \mu$ and $t$ we have

$$
\begin{aligned}
& R(\lambda) E(t)=E(t) R(\lambda) \\
& P(\lambda) E(t)=S(t) R(\lambda) \\
& R(\lambda)-R(\mu)=(\mu-\lambda) R(\lambda) R(\mu)=(\mu-\lambda) R(\mu) R(\lambda) \\
& P(\lambda)-P(\mu)=(\mu-\lambda) P(\lambda) R(\mu)=(\mu-\lambda) P(\mu) R(\lambda) .
\end{aligned}
$$

Proof. The identities (4) and (5) follow from $E(t) E(s)=E(s) E(t)$ and $S(t) E(s)=$ $S(s) E(t)$ after taking the Laplace transform with respect to $s$. The identity (6) follows from the semigroup property and (7) follows from the empathy relation (2), taking Laplace transforms with respect to $s$ and $t$. Since there are a number of different ways of deriving the last two identities, we shall indicate an effective method for deriving (7):

$$
\begin{aligned}
(\mu-\lambda) P(\lambda) R(\mu) & =(\mu-\lambda) \int_{0}^{\infty} e^{-\lambda t} S(t) \int_{0}^{\infty} e^{-\mu s} E(s) y d s d t \\
& =\int_{0}^{\infty} \frac{d}{d t} e^{(\mu-\lambda) t} \int_{0}^{\infty} e^{-\mu(s+t)} S(s+t) y d s d t .
\end{aligned}
$$

The change of variable $s \rightarrow r=s+t$ and subsequent change of the order of integration give 


$$
\begin{aligned}
(\mu-\lambda) P(\lambda) R(\mu) y & =\int_{0}^{\infty} \int_{0}^{r} \frac{d}{d t} e^{(\mu-\lambda) t} e^{-\mu r} S(r) y d r d t \\
& =\int_{0}^{\infty}\left(e^{(\mu-\lambda) r}-1\right) e^{-\mu r} S(r) y d r=P(\lambda) y-P(\mu) y
\end{aligned}
$$

We note that the proof of (7) does not rely on the invertibility condition (3). The proof of (6) is based on the semigroup property which has been derived from (2) and (3).

It is well-known that the pseudo-resolvent property (6) implies that for $\lambda$ and $\mu$ arbitrary $\operatorname{Ker} R(\lambda)=\operatorname{Ker} R(\mu)=: N_{E}$. For $P(\lambda)$ we have a more intricate property which is derived from $(7)$ :

Lemma 2.4. If (2) holds then $N_{E} \cap \operatorname{Ker} P(\lambda)=N_{E} \cap \operatorname{Ker} P(\mu)$ for every positive $\lambda$ and $\mu$.

Proof. If $y \in N_{E} \cap \operatorname{Ker} P(\mu)$ then, by (7) we have $P(\lambda) y=(\mu-\lambda) P(\lambda) R(\mu) y=0$ and hence $y \in \operatorname{Ker} P(\lambda)$.

Corollary 2.5. If (2) and (3) hold then $P(\lambda)$ is invertible for every $\lambda>0$.

Proof. Suppose that $P(\lambda) y=0$. By $(7)$ we have $P(\xi) y=(\lambda-\xi) P(\xi) R(\lambda) y$. It follows from (3) that $y=(\lambda-\xi) R(\lambda) y$ and hence $R(\xi) y=(\lambda-\xi) R(\xi) R(\lambda) y$. Comparison with (6) shows that $R(\lambda) y=0$. Hence $y \in N_{E} \cap \operatorname{Ker} P(\lambda)=N_{E} \cap \operatorname{Ker} P(\xi)=\{0\}$.

The pseudo-resolvent equation (6) implies that $R(\lambda)[Y]=R(\mu)[Y]=: \mathfrak{D}_{E} \subset Y$ for every $\lambda, \mu>0$. Likewise, (7) implies that $P(\lambda)[Y]=P(\mu)[Y]=: \mathfrak{D} \subset X$. From (4) and (5) we obtain

LEMMA 2.6. $E(t)\left[\mathfrak{D}_{E}\right] \subset \mathfrak{D}_{E}$ and $S(t)\left[\mathfrak{D}_{E}\right] \subset \mathfrak{D}$ for any $t>0$.

The "domains" $\mathfrak{D}$ and $\mathfrak{D}_{E}$ play an important role in the behaviour of $S(t)$ and $E(t)$ at $t=0$. We prepare for this in the following result:

Lemma 2.7. Let $y=R(\lambda) y_{\lambda} \in \mathfrak{D}_{E}$ where $y_{\lambda} \in Y$. Then

$$
\begin{aligned}
& E(t) y=e^{\lambda t}\left[y-\int_{0}^{t} e^{-\lambda s} E(s) y_{\lambda} d s\right], \\
& S(t) y=e^{\lambda t}\left[P(\lambda) y_{\lambda}-\int_{0}^{t} e^{-\lambda s} S(s) y_{\lambda} d s\right] .
\end{aligned}
$$

Pr o of. We shall prove (9) in some detail. The proof of (8) is similar.

$$
S(t) y=S(t) R(\lambda) y_{\lambda}=S(t) \int_{0}^{\infty} e^{-\lambda s} E(s) y_{\lambda} d s=e^{\lambda t} \int_{0}^{\infty} e^{-\lambda(t+s)} S(t+s) y_{\lambda} d s .
$$

The substitution $s \rightarrow r=t+s$ gives

$$
S(t) y=e^{\lambda t} \int_{t}^{\infty} e^{-\lambda r} S(r) y_{\lambda} d r=e^{\lambda t}\left[P(\lambda) y_{\lambda}-\int_{0}^{t} e^{-\lambda r} S(r) y d r\right]
$$

THEOREM 2.8. The following statements hold:

(a) For every $y \in \mathfrak{D}_{E}, \lim _{t \rightarrow 0+} E(t) y=y$. 
(b) There exists a linear operator $C_{0}: \mathfrak{D}_{E} \rightarrow \mathfrak{D}$ such that $\lim _{t \rightarrow 0+} S(t) y=C_{0} y$ for every $y \in \mathfrak{D}_{E}$.

(c) The operators $R(\lambda)$ are invertible for every $\lambda>0$.

(d) $C_{0}=P(\lambda) R^{-1}(\lambda)$ is invertible and $\mathfrak{R}\left(C_{0}\right)=\mathfrak{D}$.

Proof. Statement (a) is a direct consequence of (8) and (b) follows from (9). In fact, $C_{0} y=P(\lambda) y_{\lambda}$ where $y=R(\lambda) y_{\lambda}$. Suppose that $y \in \mathfrak{D}_{E}$ has the representations $y=R(\lambda) y_{\lambda}=R(\lambda) z_{\lambda}$. Then $C_{0} y=P(\lambda) y_{\lambda}=P(\lambda) z_{\lambda}$. From the invertibility of $P(\lambda)$ we see that $y_{\lambda}=z_{\lambda}$ and (c) is proved. The final statement follows after a moment of reflection.

Remark 2.9. The invertibility of the $R(\lambda)$ implies that $\mathfrak{D}_{E}$ is trivial if and only if $Y$ is trivial. Similarly the domain $\mathfrak{D}$ cannot be trivial unless $Y$ is trivial.

The behaviour of $S(t)$ and $E(t)$ at $t=0$ may also be expressed as an asymptotic property of their Laplace transforms.

TheOREM 2.10. For any $y \in \mathfrak{D}_{E}$,

$$
\lim _{\lambda \rightarrow \infty} \lambda R(\lambda) y=y, \quad \lim _{\lambda \rightarrow \infty} \lambda P(\lambda) y=C_{0} y .
$$

Proof. Let $y=R(\mu) y_{\mu}$. We apply (7) to $y_{\mu}$ to obtain $\lambda P(\lambda) y=C_{0} y+P(\lambda)\left[\mu y-y_{\mu}\right]$. From the dominated convergence theorem it is seen that $\lim _{\lambda \rightarrow \infty} P(\lambda) z=0$ for every $z \in Y$ and the result for $\lambda P(\lambda) y$ follows. The statement about $\lambda R(\lambda) y$ follows similarly from (6).

3. Bounded resolvents. All the results of the previous section were obtained without assuming boundedness of the operators $R(\lambda)$ or $P(\lambda)$. In this section it will be shown that under such additional assumptions stronger results are possible. For this purpose we introduce some subspaces of $Y$.

Let $Y_{E}$ be the closure of $\mathfrak{D}_{E}$ in $Y$ and set $\mathfrak{D}_{E, 1}=R(\lambda)\left[Y_{E}\right], \mathfrak{D}_{E, 2}=R(\lambda)\left[\mathfrak{D}_{E}\right]$. From (6) it is seen that the last two subspaces are independent of the choice of $\lambda$. We also define the space of attractors of $\mathcal{E}$ by $\operatorname{Att}(\mathcal{E})=\left\{y \in Y \mid \lim _{t \rightarrow 0+} E(t) y\right.$ exists. $\}$

From Theorems 2.8 and 2.10 we see that $\mathfrak{D}_{E, 2} \subset \mathfrak{D}_{E, 1} \subset \mathfrak{D}_{E} \subset Y_{E}$ and the inclusions are dense. Moreover, $\mathfrak{D}_{E} \subset \operatorname{Att}(\mathcal{E})$. In order to be able to say more about $\operatorname{Att}(\mathcal{E})$, we need the following result:

Lemma 3.1. Let $\langle\mathcal{S}, \mathcal{E}\rangle$ be an empathy. For any $y \in Y$ and $t>0$ it is true that $\int_{0}^{t} e^{-\lambda s} E(s) y d s \in \mathfrak{D}_{E}$ and $\int_{0}^{t} e^{-\lambda s} S(s) y d s \in \mathfrak{D}$. Furthermore,

$$
E(t) y=e^{\lambda t}\left[y-R^{-1}(\lambda) \int_{0}^{t} e^{-\lambda s} E(s) y d s\right]=e^{\lambda t}\left[y-P^{-1}(\lambda) \int_{0}^{t} e^{-\lambda s} S(s) y d s\right] .
$$

Proof. From the empathy relation $S(s) E(t) y=S(t+s) y$ we obtain

$$
P(\lambda) E(t) y=\int_{0}^{\infty} e^{-\lambda s} S(t+s) y d s=e^{\lambda t} \int_{t}^{\infty} e^{-\lambda s} S(s) y d s=e^{\lambda t}\left[P(\lambda) y-\int_{0}^{t} e^{-\lambda s} S(s) y d s\right] .
$$


In the same way, starting from the semigroup property, we can prove that

$$
R(\lambda) E(t) y=e^{\lambda t}\left[R(\lambda) y-\int_{0}^{t} e^{-\lambda s} E(s) y d s\right] .
$$

The final conclusion is reached after a little thought on the two identities.

TheOrem 3.2. If $P(\lambda)$ or $R(\lambda)$ is bounded for some $\lambda>0$ then $\operatorname{Att}(\mathcal{E}) \subset Y_{E}$, and the inclusion is dense. Furthermore, if $y \in \operatorname{Att}(\mathcal{E})$, then $\lim _{t \rightarrow 0+} E(t) y=y$.

P r o of. Suppose that $P(\lambda)$ is bounded. Then $P^{-1}(\lambda)$ is closed. If $y \in \operatorname{Att}(\mathcal{E})$, we see from (10) that $\lim _{t \rightarrow 0+} P^{-1}(\lambda) \int_{0}^{t} e^{-\lambda s} S(s) y d s$ exists. Since the integral tends to zero, it follows that $\lim _{t \rightarrow 0+} E(t) y=y$. It remains to be proved that $y \in Y_{E}$ and this is done by observing that $\mu R(\mu) y=\int_{0}^{\infty} e^{-t} E(t / \mu) y d t$. A straightforward application of the dominated convergence theorem shows that $\lim _{\mu \rightarrow \infty} \mu R(\mu) y=y$, which implies that $y \in Y_{E}$. The case where $R(\lambda)$ is bounded is treated in exactly the same way.

Remark 3.3. Theorem 3.2 holds for semigroups which are unrelated to a family $\mathcal{S}$ of evolution operators but regarded as if "in empathy with themselves". This means that the Laplace transforms $R(\lambda) y$ are assumed to exist and that $R(\xi)$ is assumed to be invertible for some $\xi>0$.

From Theorem 2.10 we obtain directly

TheOREm 3.4. Assume that the operators $P(\lambda)$ are bounded for every $\lambda>0$ and $\|\lambda P(\lambda)\|=O(1)$ as $\lambda \rightarrow \infty$. Then the operator $C_{0}$ is bounded and for its extension $C$ to $Y_{E}$ it is true that $C y=\lim _{\lambda \rightarrow \infty} \lambda P(\lambda) y$.

THEOREM 3.5. Under the hypotheses of Theorem 3.4 the operator $C$ is invertible if and only if $C_{0}^{-1}$ is closeable.

Proof. If $C$ is invertible, its inverse is closed and therefore $C_{0}^{-1}$, being the restriction of a closed operator, is closable. Conversely, assume that $C_{0}^{-1}$ is closable and suppose that $C y=0$. Let $\left\{y_{n}\right\} \subset \mathfrak{D}_{E}$ be such that $y_{n} \rightarrow y$. Then $x_{n}:=C y_{n}=C_{0} y_{n} \rightarrow 0$ and $y_{n}=C_{0}^{-1} x_{n} \rightarrow y$ and hence $y=0$.

4. The Post-Widder inversion theorem. Let $g: t \in(0, \infty) \rightarrow g(t) \in Z$ be a function with values in some Banach space $Z$, and suppose that the Laplace transform $f(\lambda)$ exists for any $\lambda>0$. Then it is well-known that $f \in C^{\infty}(0, \infty)$ and $f^{(k)}(\lambda)=$ $(-1)^{k} \int_{0}^{\infty} t^{k} e^{-\lambda t} g(t) d t$ (see [HP57, p. 216]). The Widder operators $L_{k, t} f$ are defined by

$$
L_{k, t} f=\frac{(-1)^{k}}{k !}\left(\frac{k}{t}\right)^{k+1} f^{(k)}\left(\frac{k}{t}\right), \quad t>0, k=1,2, \ldots
$$

The Post-Widder inversion theorem states that for $t$ in the Lebesgue set of $g$, $\lim _{k \rightarrow \infty}\left\|L_{k, t} f-g(t)\right\|=0$ and the convergence is uniform on any compact subinterval of an interval on which $g$ is continuous [HP57, Theorem 6.3.5, p. 224; Wid46, Theorem 6a, p. 288; Theorem 5a, p. 285]. 
If we apply this method of approximation to the empathy $\langle\mathcal{S}, \mathcal{E}\rangle$ we obtain the following expressions:

$$
L_{k, t} R(\cdot) y=\left[\frac{k}{t} R\left(\frac{k}{t}\right)\right]^{k+1} y, \quad L_{k, t} P(\cdot) y=\left[\frac{k}{t} P\left(\frac{k}{t}\right)\right]\left[\frac{k}{t} R\left(\frac{k}{t}\right)\right]^{k} y,
$$

for $y \in Y$. We have made use of (6) and (7) to derive the expressions $R^{(k)}(\lambda) y=$ $(-1)^{k} k ! R^{k+1}(\lambda) y$ and $P^{(k)}(\lambda) y=(-1)^{k} k ! P(\lambda) R^{k}(\lambda) y$. Because of the strong continuity of $\mathcal{E}$ and $\mathcal{S}$ (Theorem 2.2) the Post-Widder theorem has the following form:

ThEOREM 4.1. Let $\langle\mathcal{S}, \mathcal{E}\rangle$ be an empathy, then for every $t>0$ and every $y \in Y$,

$$
E(t) y=\lim _{k \rightarrow \infty} L_{k, t} R(\cdot) y, \quad S(t) y=\lim _{k \rightarrow \infty} L_{k, t} P(\cdot) y .
$$

The convergence is uniform on compact subintervals of $(0, \infty)$.

Since $L_{k, t} R(\cdot) y \in \mathfrak{D}_{E}$ and $L_{k, t} P(\cdot) y \in \mathfrak{D}$ we have

Corollary 4.2. Let $X_{S}$ be the closure of $\mathfrak{D}$ in $X$. Then $E(t)[Y] \subset Y_{E}$ and $S(t)[Y] \subset$ $X_{S}$ for every $t>0$.

THEOREM 4.3. Assume that the operators $P(\lambda)$ are bounded and $\|\lambda P(\lambda)\|=O(1)$ as $\lambda \rightarrow \infty$. Then $S(t)=C E(t)$.

Proof. By Theorem 2.8 (d) we have $C R(\lambda) y=C_{0} R(\lambda) y=P(\lambda) y$ for any $y \in Y$. Since $C$ is bounded we see by virtue of Corollary 4.2 that $\int_{0}^{\infty} e^{-\lambda t} C E(t) y d t=C R(\lambda) y=$ $\int_{0}^{\infty} e^{-\lambda t} S(t) y d t$ for every $\lambda>0$. The result follows from the uniqueness of the Laplace transform [Wid46, Theorem 6.3, p. 63].

Remark 4.4. If we restrict $\mathcal{S}$ and $\mathcal{E}$ to $Y_{E}$ the restriction $\left\langle\mathcal{S}_{1}, \mathcal{E}_{1}\right\rangle$ is an empathy with $X$ and $Y$ replaced by $X_{S}$ and $Y_{E}$ respectively, $\mathfrak{D}$ replaced by $\mathfrak{D}_{1}=P(\lambda)\left[Y_{E}\right]$ and $\mathfrak{D}_{E}$ replaced by $\mathfrak{D}_{E, 1}$.

5. The generator of an empathy. Let us define the operator $B: \mathfrak{D} \rightarrow \mathfrak{D}_{E}$ as $B=$ $C_{0}^{-1}=R(\lambda) P^{-1}(\lambda)$. By Theorem 2.8 this definition is possible and the representation is independent of $\lambda$. Set $A_{\lambda}=[\lambda R(\lambda)-I] P^{-1}(\lambda): \mathfrak{D} \rightarrow Y$.

Proposition 5.1. $A_{\lambda}=A_{\mu}=: A$, and $P(\lambda)=(\lambda B-A)^{-1}$ for arbitrary $\lambda, \mu>0$.

Proof. Inversion of $(7)$ gives $P^{-1}(\mu)-P^{-1}(\lambda)=(\mu-\lambda) R(\mu) P^{-1}(\lambda)=(\mu-$ $\lambda) R(\lambda) P^{-1}(\lambda)=(\mu-\lambda) B$. Therefore, $A_{\lambda}-A_{\mu}=\lambda B-P^{-1}(\lambda)-\left[\mu B-P^{-1}(\mu)\right]=$ $(\lambda-\mu) B-\left[P^{-1}(\lambda)-P^{-1}(\mu)\right]=0$.

The operator pair $\langle A, B\rangle$ is called the generator of the empathy $\langle\mathcal{S}, \mathcal{E}\rangle$. From the uniqueness of the Laplace transform it is clear that the assignment $\langle\mathcal{S}, \mathcal{E}\rangle \rightarrow\langle A, B\rangle$ is bi-unique.

From Theorem 2.8 we see that $R(\lambda)$ is the resolvent of some operator $A_{E}: \mathfrak{D}_{E} \rightarrow Y_{E}$, i.e. $R(\lambda)=\left(\lambda I-A_{E}\right)^{-1}$. The operator $A_{E}$ is called the generator of the semigroup $\mathcal{E}$. Making use of the fact that $A_{E}=\lambda I-R^{-1}(\lambda)$ it is easily seen that $A_{E}=A B^{-1}=A C_{0}$.

Next we investigate the relationship between the generator $\langle A, B\rangle$ and the Cauchy problem (1). 
TheOREM 5.2. Let $\langle A, B\rangle$ be the generator of the empathy $\langle\mathcal{S}, \mathcal{E}\rangle$. For given $y \in \mathfrak{D}_{E}$ the function $u: t \rightarrow u(t)=S(t) y$ is a solution of the Cauchy problem (1). Moreover,

$$
\frac{d}{d t}[B S(t) y]=A S(t) y=B S(t) A C_{0} y .
$$

Proof. From Lemma 2.6 we see that $u(t) \in \mathfrak{D}$. It follows from Theorem $2.8(\mathrm{~d}),(4)$ and $(5)$ that $v(t):=B u(t)=B S(t) y=E(t) y$. Since $\mathcal{E}$ is strongly continuous on $(0, \infty)$ we infer from $(8)$ that $v(t)$ is differentiable on $(0, \infty)$. Indeed,

$$
\begin{aligned}
\frac{d v}{d t} & =\lambda E(t) y-E(t) R^{-1}(\lambda) y=E(t)\left[\lambda y-R^{-1}(\lambda) y\right]=\left[\lambda I-R^{-1}(\lambda)\right] E(t) y \\
& =E(t) A_{E} y=A_{E} E(t) y=B S(t) A B^{-1} y=A B^{-1} B S(t) y=A S(t) y
\end{aligned}
$$

The initial condition is satisfied by virtue of Theorem $2.8(\mathrm{a})$.

It is instructive to compare the generator $A_{E}$ of $\mathcal{E}$ with its infinitesimal operator/generator $A_{0}$ which is defined in the usual way: Let $A_{h} y=h^{-1}[E(h) y-y]$. Then $y \in Y$ is in $\mathfrak{D}\left(A_{0}\right)$ if $\lim _{h \rightarrow 0+} A_{h} y=: A_{0} y$ exists. We immediately see that $\mathfrak{D}\left(A_{0}\right) \subset$ $\operatorname{Att}(\mathcal{E})$. Indeed, if $y \in \mathfrak{D}\left(A_{0}\right)$ then $\lim _{h \rightarrow 0+} E(h) y=y$. Let $\mathfrak{D}_{E}^{1}=R(\lambda)[\operatorname{Att}(\mathcal{E})] \subset \mathfrak{D}_{E}$.

TheOREM 5.3. Suppose that $R(\lambda)$ is bounded for some $\lambda>0$. Then $\mathfrak{D}_{E}^{1} \subset \mathfrak{D}\left(A_{0}\right) \subset$ $\mathfrak{D}_{E}$ and $A_{0}=A_{E}$ in $\mathfrak{D}\left(A_{0}\right)$. In addition $A_{0}$ is closable.

Proof. Let $y \in \mathfrak{D}\left(A_{0}\right)$. From (10) we see that

$$
A_{h} y=h^{-1}\left[e^{\lambda h}-1\right] y-e^{\lambda h} R^{-1}(\lambda) h^{-1} \int_{0}^{h} e^{-\lambda s} E(s) y d s .
$$

Since the limits of the first two terms in (11) exist, it follows that

$$
\lim _{h \rightarrow 0+} R^{-1}(\lambda) h^{-1} \int_{0}^{h} e^{-\lambda s} E(s) y d s
$$

exists. Also since $y \in \operatorname{Att}(\mathcal{E})$ we have $\lim _{h \rightarrow 0+} h^{-1} \int_{0}^{h} e^{-\lambda s} E(s) y d s=y$ and hence the closedness of $R^{-1}(\lambda)$ implies that $y \in \mathfrak{D}_{E}$ and $A_{0} y=\lambda y-R^{-1}(\lambda) y=A_{E} y$.

Let $y \in \mathfrak{D}_{E}^{1}$ be represented as $y=R(\lambda) y_{\lambda}$ with $y_{\lambda} \in \operatorname{Att}(\mathcal{E})$. From (8) we obtain

$$
A_{h} y=h^{-1}\left[e^{\lambda h}-1\right] y-e^{\lambda h} h^{-1} \int_{0}^{h} e^{-\lambda s} E(s) y_{\lambda} d s .
$$

The terms on the right hand side of (12) are both convergent as $h \rightarrow 0$ and it follows that $y \in \mathfrak{D}\left(A_{0}\right)$.

Finally, since $A_{E}=\lambda I-R^{-1}(\lambda)$ is closed, it follows that $A_{0}$ is closable.

A number of results obtained so far required that either $R(\lambda)$ or $P(\lambda)$ be bounded for some $\lambda>0$. These assumptions imply boundedness of the resolvents $R(\mu)$ and $P(\mu)$ and the closedness of the generator $\langle A, B\rangle$ of the underlying empathy.

THEOREM 5.4. If for some $\lambda>0$ both $P(\lambda)$ and $R(\lambda)$ are bounded, then $P(\mu)$ and $R(\mu)$ are bounded for every $\mu>0$. Moreover, the operator $\langle A, B\rangle: x \in \mathfrak{D} \rightarrow\langle A x, B x\rangle \in Y \times Y$ is closed. 
Proof. As seen in the proof of Theorem 5.3, $A_{E}$ is closed. Therefore $R^{-1}(\mu)=$ $\mu I-A_{E}$ is closed and hence $R(\mu)$ is bounded according to the closed graph theorem. From (7) we see that $P(\mu)=P(\lambda)+(\lambda-\mu) P(\lambda) R(\mu)$ is bounded. Since $\mu B-A$ is closed for at least two values of $\mu$ it is easily proved that the operator $\langle A, B\rangle$ is closed.

6. Uniqueness. In the previous section we have shown that if $\langle A, B\rangle$ is the generator of an empathy $\langle\mathcal{S}, \mathcal{E}\rangle$ and $y \in \mathfrak{D}_{E}$, then $u(t)=S(t) y$ is a solution of (1). In this section we shall describe a class of functions $L_{S E}$ which is such that any solution of (1) which is in this class, has the representation $u(t)=S(t) y$. If $u: t \in(0, \infty) \rightarrow u(t)$ is a function for which the Laplace transform exists we shall write $\widehat{u}(\lambda)=\int_{0}^{\infty} e^{-\lambda t} u(t) d t$. Suppose that $u: t \rightarrow u(t) \in \mathfrak{D} \subset X$ so that the representation $u(t)=P(\lambda) v_{\lambda}(t)$ is valid. We say that $u$ is of class $L_{S E}$ if for some $\lambda>0, v_{\lambda}$ has a Laplace transform in $Y$.

LEMMA 6.1. If $u$ is of class $L_{S E}$, the Laplace transforms of $u$ and $R(\lambda) v_{\lambda}$ exist. Moreover, $\widehat{u}(\mu) \in \mathfrak{D}, \widehat{R(\lambda)} v_{\lambda}(\mu) \in \mathfrak{D}_{E}$ and

$$
\widehat{u}(\mu)=P(\lambda) \widehat{v_{\lambda}}(\mu) ; \quad R \widehat{R(\lambda)} v_{\lambda}(\mu)=R(\lambda) \widehat{v_{\lambda}}(\mu) .
$$

Proof. Since the operators $S(t)$ are bounded

$$
P(\lambda) \widehat{v_{\lambda}}(\mu)=\int_{0}^{\infty} e^{-\lambda t} S(t) \int_{0}^{\infty} e^{-\mu s} v_{\lambda}(s) d s d t=\int_{0}^{\infty} e^{-\lambda t} \int_{0}^{\infty} e^{-\mu s} S(t) v_{\lambda}(s) d s d t .
$$

An application of Fubini's theorem shows that the right hand side equals $P \widehat{(\lambda)} v_{\lambda}(\mu)=$ $\widehat{u}(\mu)$. A similar calculation on $R(\lambda) \widehat{v_{\lambda}}(\mu)$ concludes the proof.

Remark 6.2. From (7) we see that $v_{\mu}(t)-v_{\lambda}(t)=(\mu-\lambda) R(\lambda) v_{\lambda}(t)$, which shows that the definition of class $L_{S E}$ is independent of the choice of $\lambda$.

Lemma 6.3. Let $y \in \mathfrak{D}_{E}$. Then $u: t \rightarrow S(t) y$ is of class $L_{S E}$.

Proof. By $(5), u(t)=S(t) R(\lambda) y_{\lambda}=P(\lambda) E(t) y_{\lambda}$, if $y=R(\lambda) y_{\lambda}$. Thus $v_{\lambda}=E(t) y_{\lambda}$ and the proof is complete.

LEMMA 6.4. Let $\langle A, B\rangle$ be the generator of an empathy. If $u$ is of class $L_{S E}$ then the Laplace transforms of $A u$ and $B u$ exist. In particular, $\widehat{A u}=A \widehat{u}$ and $\widehat{B u}=B \widehat{u}$.

Proof. $B u(t)=R(\lambda) P^{-1}(\lambda) u(t)=R(\lambda) v_{\lambda}(t)$ and $A u(t)=\lambda R(\lambda) v_{\lambda}(t)-v_{\lambda}(t)$. The result follows from Lemma 6.1 .

THEOREM 6.5. Let $\langle A, B\rangle$ be the generator of the empathy $\langle\mathcal{S}, \mathcal{E}\rangle$. If $u$ is of class $L_{S E}$ and solves the Cauchy problem (1) with $y \in \mathfrak{D}_{E}$ then $u(t)=S(t) y$.

Proof. Let $w(t)=S(t) y$. Then $\widehat{w}(\mu)=P(\mu) y$. Since $u$ is of class $L_{S E}$, the Laplace transform of $A u$ exists and therefore the Laplace transform of the derivative of $B u$ exists. Taking the Laplace transform in (1) is therefore justified and from Lemma 6.4 we obtain $\mu B \widehat{u}(\mu)-y=A \widehat{u}(\mu)$. Thus $\widehat{u}(\mu)=P(\mu) y=\widehat{w}(\mu)$ for arbitrary $\mu$. By the uniqueness of the Laplace transform, $u(t)=w(t)$.

Rem ark 6.6. Note that the uniqueness theorem was obtained without assumptions on the boundedness of $P(\lambda)$ or $R(\lambda)$. 
R e mark 6.7. If the semigroup $\mathcal{E}$ is of class $\left(C_{0}\right)$ the uniqueness is unconditional, for if we set $v(t)=B u(t)$, then

$$
\frac{d v}{d t}=A u(t)=A B^{-1} v(t)=A_{E} v(t)=A_{0} v(t) .
$$

In this case it is well-known that $v(t)=E(t) y=B S(t) y$ and hence $u(t)=S(t) y$. If $\mathcal{E}$ is not of class $\left(C_{0}\right)$, the argument that $v(t)=E(t) y$ does not extend since the norms $\|E(t)\|$ need not be bounded on intervals of the form $(0, a]$.

7. Uniformly bounded empathies. The empathy $\langle\mathcal{S}, \mathcal{E}\rangle$ is called uniformly bounded if there exist positive constants $M^{\prime}$ and $N^{\prime}$ such that $\|S(t)\| \leq M^{\prime}$ and $\|E(t)\| \leq$ $N^{\prime}$ for every $t>0$.

ThEOREM 7.1. The empathy $\langle\mathcal{S}, \mathcal{E}\rangle$ is uniformly bounded if and only if the resolvent operators $P(\lambda)$ and $R(\lambda)$ are bounded and there exist positive constants $M$ and $N$ such that for any $\lambda>0$ and any $k=1,2, \ldots$,

$$
\|\lambda P(\lambda)\| \leq M \quad \text { and } \quad\left\|\lambda^{k} R^{k}(\lambda)\right\| \leq N .
$$

Pr o of. Suppose the empathy is uniformly bounded. The boundedness of the resolvent operators are obtained directly and the first of the inequalities (14) come from the same effort. The second inequality follows from the identity

$$
R^{k+1}(\lambda) y=\frac{1}{k !} \int_{0}^{\infty} e^{-\lambda t} t^{k} E(t) y d t,
$$

which is obtained with the aid of the convolution theorem for Laplace transforms.

Suppose, conversely, that the resolvent operators are bounded and (14) holds. We then conclude for the Widder operators $L_{k, t}$ that

$$
\left\|L_{k, t} R(\cdot) y\right\| \leq N\|y\| \quad \text { and } \quad\left\|L_{k, t} P(\cdot) y\right\| \leq M N\|y\| .
$$

It follows from Theorem 4.1 that $\|S(t)\| \leq M N$ and $\|E(t)\| \leq N$ for any $t>0$.

THEOREM 7.2. If $\langle\mathcal{S}, \mathcal{E}\rangle$ is uniformly bounded then the semigroup $\mathcal{E}_{1}$ is of class $\left(C_{0}\right)$ with generator $A_{E, 1}=A C_{1}$ where $C_{1}$ is the restriction of $C$ to $\mathfrak{D}_{1}=R(\lambda)\left[Y_{E}\right]$.

Proof. Because of the uniform boundedness, $\operatorname{Att}(\mathcal{E})$ is closed and therefore $Y_{E}=$ $\operatorname{Att}(\mathcal{E})$ (Theorem 3.2). Hence $\mathcal{E}_{1}$ is of class $\left(C_{0}\right)$. The rest is evident.

8. Generation of uniformly bounded empathies. We now turn to the more profound question of constructing from a given pair $\langle A, B\rangle$ of linear operators an empathy $\langle\mathcal{S}, \mathcal{E}\rangle$ having $\langle A, B\rangle$ as its generator.

Let $X$ and $Y$ be Banach spaces and $A$ and $B$ linear operators with a common domain $\mathfrak{D} \subset X$ and values in $Y$. We shall assume that $\lambda B-A$ is invertible for every $\lambda>0$. Let the inverse be denoted by $P(\lambda): Y \rightarrow \mathfrak{D}$. We set $R(\lambda)=B P(\lambda)$. A straightforward calculation shows that

$$
P(\lambda)-P(\mu)=(\mu-\lambda) P(\lambda) B P(\mu)=(\mu-\lambda) P(\lambda) R(\mu)=(\mu-\lambda) P(\mu) R(\lambda)
$$

and it follows that

$$
R(\lambda)-R(\mu)=(\mu-\lambda) R(\lambda) R(\mu)=(\mu-\lambda) R(\mu) R(\lambda) .
$$


We define the domain $\mathfrak{D}_{E}:=B[\mathfrak{D}]=R(\lambda)[Y]$ and the subspace $Y_{E} \subset Y$ as the closure of $\mathfrak{D}_{E}$ in $Y$.

TheOREM 8.1. If $\lim _{\lambda \rightarrow \infty} P(\lambda) z=0$ for every $z \in Y$ then $B$ is invertible and $B^{-1} y=\lim _{\lambda \rightarrow \infty} \lambda P(\lambda) y$ for every $y \in \mathfrak{D}_{E}$. Conversely, if $B^{-1}$ exists and $B^{-1} y=$ $\lim _{\lambda \rightarrow \infty} \lambda P(\lambda) y$ then $\lim _{\lambda \rightarrow \infty} P(\lambda) z=0$ for every $z \in \mathfrak{R}(A)$. In either case $R(\mu)$ is invertible for any $\mu>0$.

Proof. The proof of the first statement is contained in the proof of Theorem 2.10 based on the identity

$$
\lambda P(\lambda) y=P(\lambda)\left[\mu y-y_{\mu}\right]+P(\mu) y_{\mu}
$$

where $R(\mu) y_{\mu}=y \in \mathfrak{D}_{E}$. The same argument also yields the invertibility of $R(\mu)$.

To prove the second statement, we note that under the hypotheses, $R(\mu)$ is invertible for arbitrary $\mu$ and $B^{-1}=P(\mu) R^{-1}(\mu)$. Moreover, $R^{-1}(\mu)=\mu I-A B^{-1}$. Hence $y_{\mu}=$ $R^{-1}(\mu) y=\mu y-A B^{-1} y$ and (16) may be rewritten in the form $\lambda P(\lambda) y=P(\lambda) A B^{-1} y+$ $B^{-1} y$. This concludes the proof.

TheOREM 8.2. Suppose the space $Y$ has the Radon-Nikodym property. The operator pair $\langle A, B\rangle$ is the generator of a uniformly bounded empathy $\langle\mathcal{S}, \mathcal{E}\rangle$ if and only if the operators $P(\lambda)$ and $R(\lambda)$ are bounded for every $\lambda>0$ and there exist positive numbers $M$ and $N$ such that for every $\lambda>0$ and $k=1,2, \ldots$,

$$
\|\lambda P(\lambda)\| \leq M \quad \text { and } \quad\left\|\lambda^{k} R^{k}(\lambda)\right\| \leq N .
$$

Pr o of. The necessity part is contained in Theorem 7.1. Suppose that (17) holds. By Theorem 8.1 the operator $B$ is invertible. Since $\|\lambda P(\lambda)\| \leq M$, it follows that there exists a bounded linear operator $C: Y_{E} \rightarrow X$ with $\|C\| \leq M$ such that $B^{-1}$ is the restriction of $C$ to $\mathfrak{D}_{E}$. We shall use the following notation: $\mathfrak{D}_{E, 1}:=R(\lambda)\left[Y_{E}\right] ; \mathfrak{D}_{1}:=B^{-1}\left[\mathfrak{D}_{E, 1}\right]$; $\left\langle A_{1}, B_{1}\right\rangle$ the restriction of $\langle A, B\rangle$ to $\mathfrak{D}_{1} ; R_{1}(\lambda)$ and $P_{1}(\lambda)$ the restrictions of $R(\lambda)$ and $P(\lambda)$ to $Y_{E}$ respectively.

By the Hille-Yosida Theorem and Theorem 7.1 there exists a semigroup $\mathcal{E}_{1}$ of class $\left(C_{0}\right)$ on $Y_{E}$ such that $R_{1}(\lambda) y$ is the Laplace transform of $E_{1}(\cdot) y$ for every $y \in Y_{E}$. The generator of $\mathcal{E}_{1}$ is $A_{1} B_{1}^{-1}$ which is also the infinitesimal generator. If we define the family $\mathcal{S}_{1}=\left\{S_{1}(t)=C E_{1}(t) \mid t>0\right\}$ it is easy to see that $\left\langle\mathcal{S}_{1}, \mathcal{E}_{1}\right\rangle$ is an empathy with generator $\left\langle A_{1}, B_{1}\right\rangle$.

From (15) and (17) we see that the function $\lambda \rightarrow R(\lambda) y$ is infinitely differentiable so that the Widder operators $L_{k, t} R(\cdot) y$ are defined and bounded. In fact, by (17), $\left\|L_{k, t} R(\cdot) y\right\| \leq N\|y\|$ for any $y \in Y$. Since $Y$ has the Radon-Nikodým property, Widder's inversion theorem [Wid46, Theorem 16a, p. 315; Theorem 16b, p. 316] holds for functions with values in $Y$ [Are87] and there exists a family $\mathcal{E}=\{E(t) \mid t>0\}$ such that $\|E(t)\| \leq N$ and

$$
R(\lambda) y=\int_{0}^{\infty} e^{-\lambda t} E(t) y d t .
$$

We proceed to prove that $\mathcal{E}$ is a semigroup and an extension of $\mathcal{E}_{1}$. Firstly, since $R_{1}(\lambda) y=R(\lambda) y$ for $y \in Y_{E}$, it follows from (18), the strong continuity of $\mathcal{E}_{1}$ and the 
uniqueness of the Laplace transform, that $E(t) y=E_{1}(t) y$ for $y \in Y_{E}$. Secondly, we can use (15), noting that $R(\mu) R(\lambda)=R_{1}(\mu) R(\lambda)$, to reverse the calculation leading to (6) and obtain for any fixed $y \in Y$,

$$
\int_{0}^{\infty} e^{-\lambda t} E_{1}(t) \int_{0}^{\infty} e^{-\mu s} E(s) y d s d t=\int_{0}^{\infty} e^{-\lambda t} \int_{0}^{\infty} e^{-\mu s} E(t+s) y d s d t
$$

which is valid for all $\lambda, \mu>0$. From the uniqueness of the Laplace transform and the strong continuity of $\mathcal{E}_{1}$, it follows that for all $t>0$,

$$
E_{1}(t) \int_{0}^{\infty} e^{-\mu s} E(s) y d s=\int_{0}^{\infty} e^{-\mu s} E(t+s) y d s
$$

for arbitrary $\mu>0$. By the Post-Widder inversion theorem, $E(s) y \in Y_{E}$ for $s$ in the Lebesgue set of $E(\cdot) y$. Hence the operator $E_{1}(t)$ may be interchanged with the integral in (19) and we obtain

$$
E(t+s) y=E_{1}(t) E(s) y \quad \text { for all } t>0 \text { and } s \notin N_{y}
$$

where $N_{y} \subset(0, \infty)$ is of measure zero. Suppose that $s \notin N_{y}$. Then, by (20) we obtain $E(t+r+s) y=E_{1}(t+r) E(s) y=E_{1}(t) E_{1}(r) E(s) y=E_{1}(t) E(r+s) y$ for arbitrary $r>0$ and it follows that $r+s \notin N_{y}$. But then $N_{y}$ is empty so that $\mathcal{E}$ is indeed a semigroup and $E(t) y \in Y_{E}$ for any $t>0$ and $y \in Y$.

Next we define the family $\mathcal{S}=\{S(t)=C E(t) \mid t>0\}$ of linear operators from $Y$ to $X$. Evidently $\langle\mathcal{S}, \mathcal{E}\rangle$ is an empathy and $\|S(t)\| \leq M N$ so that it is uniformly bounded. Also,

$$
\int_{0}^{\infty} e^{-\lambda t} S(t) y d t=C R(\lambda) y=B^{-1} R(\lambda) y=P(\lambda) y .
$$

Therefore $\langle A, B\rangle$ is the generator of $\langle\mathcal{S}, \mathcal{E}\rangle$.

Remark 8.3. That $\mathcal{E}$ is a semigroup is proved in [Are87, Theorem 6.2] by making use of the notion of integrated semigroups. The proof above leans on the Post-Widder inversion theorem.

Rem ark 8.4. If $\mathfrak{D}_{E}$ is dense in $Y$, the extension procedure is unnecessary and in that case the Radon-Nikodým property is not required. This is the case if $Y$ is reflexive, for then the uniform boundedness of $\|\lambda R(\lambda)\|$ implies that $\mathfrak{D}_{E}$ is dense in $Y$.

9. An application. As a simple application of Theorem 8.2 we consider logitudinal vibrations in an elastic bar which in its reference configuration has length $l$ and linear density $\rho=\rho(x), 0 \leq x \leq l$. The equations of motion may be written as

$$
\rho(x) v_{t}(x, t)=f_{x}(x, t), \quad \sigma f_{t}(x, t)=v_{x}(x, t),
$$

where $v$ denotes the velocity, $f$ the internal force and $\sigma>0$ the reciprocal of Hooke's constant. The variable $x \in[0, l]$ denotes position in the reference configuration and $t \geq 0$ time. This particular form of the wave equation was suggested in [Fri58, p. 338]. The boundary condition at $x=0$ is simply $v(0+, t)=0$. At the other edge we assume that a particle of mass $m$ is attached so that the boundary condition becomes 


$$
m \frac{d}{d t} \gamma_{0} v=-\gamma_{0} f
$$

with $\gamma_{0} v=v(l-, t)$. For the sake of not becoming too technical we shall assume that $\rho$ is positive and continuous on $[0, l]$ so that there exists $r>0$ such that $\rho(x) \geq r$ for every $x \in[0, l]$.

The system of equations (21), (22) may be written in the form $\left(1_{1}\right)$ in the following way: Let $X=L^{2}(0, l) \times L^{2}(0, l)$ with the inner product defined as $\left(\left\langle f_{1}, g_{1}\right\rangle,\left\langle f_{2}, g_{2}\right\rangle\right)=$ $\left(f_{1}, f_{2}\right)+\left(g_{1}, g_{2}\right)$ where $($,$) denotes the usual inner product. The domain \mathfrak{D} \subset X$ is defined as $\mathfrak{D}=\left\{\langle f, g\rangle \mid f, g \in H^{1}(0, l)\right.$ and $\left.f(0+)=0\right\}$. The space $Y$ is chosen as $Y=L^{2}(0, l) \times L^{2}(0, l) \times \mathbb{R}$ where $\mathbb{R}$ denotes the reals. The elements of $Y$ will be written in the form $\langle\rho a, \sigma b, m c\rangle=:\langle\langle a, b, c\rangle\rangle$ with $a, b \in L^{2}(0, l)$ and $c \in \mathbb{R}$. The inner product in $Y$ is defined as

$$
\left[\left\langle\left\langle a_{1}, b_{1}, c_{1}\right\rangle\right\rangle,\left\langle\left\langle a_{2}, b_{2}, c_{2}\right\rangle\right\rangle\right]=\left(\rho a_{1}, a_{2}\right)+\sigma\left(b_{1}, b_{2}\right)+m c_{1} c_{2} .
$$

The associated norm will be denoted by $\llbracket \rrbracket$. The linear operators $A$ and $B$ are defined as

$$
A\langle v, f\rangle=\left\langle f_{x}, v_{x},-\gamma_{0} f\right\rangle, \quad B\langle v, f\rangle=\left\langle\rho v, \sigma f, m \gamma_{0} v\right\rangle=\left\langle\left\langle v, f, \gamma_{0} v\right\rangle\right\rangle,
$$

for $\langle v, f\rangle \in \mathfrak{D}$. To be able to apply Theorem 8.2 we have to study the equation

$$
(\lambda B-A)\langle v, f\rangle=\langle\langle a, b, c\rangle\rangle \in Y .
$$

for $\lambda>0$, which is the same as the following boundary condition problem for a system of ordinary differential equations:

$$
\begin{aligned}
& \lambda \rho v-f_{x}=\rho a, \\
& \lambda \sigma f-v_{x}=\sigma b, \\
& \lambda m \gamma_{0} v+\gamma_{0} f=m c, \\
& \left.v\right|_{x=0}=0 .
\end{aligned}
$$

Existence and uniqueness for (24) may be proved in a number of ways which we shall not describe here. We proceed to study the linear operator $P(\lambda):\langle\langle a, b, c\rangle\rangle \rightarrow\langle v, f\rangle$. To this end we notice that (23) may be written in the form

$$
\lambda\left\langle\left\langle v, f, \gamma_{0} v\right\rangle\right\rangle-\left\langle\left\langle f_{x} / \rho, v_{x} / \sigma,-\gamma_{0} f / m\right\rangle=\langle\langle a, b, c\rangle\rangle .\right.
$$

Taking the scalar product in $(25)$ with $\left\langle\left\langle v, f, \gamma_{0} v\right\rangle\right.$ yields

$$
\lambda \llbracket\left\langle\left\langle v, f, \gamma_{0} v\right\rangle\right\rangle \rrbracket^{2}-\left(f_{x}, v\right)-\left(v_{x}, f\right)+\gamma_{0} f \cdot \gamma_{0} v=\left[\langle\langle a, b, c\rangle\rangle,\left\langle\left\langle v, f, \gamma_{0} v\right\rangle\right\rangle\right] .
$$

Integration by parts of $\left(v_{x}, f\right)$ gives cancellations which lead to

$$
\lambda \llbracket\left\langle\left\langle v, f, \gamma_{0} v\right\rangle\right\rangle \rrbracket^{2}=\left[\langle\langle a, b, c\rangle\rangle,\left\langle\left\langle v, f, \gamma_{0} v\right\rangle\right\rangle\right] \leq \llbracket\langle\langle a, b, c\rangle\rangle \rrbracket \cdot \llbracket\left\langle\left\langle v, f, \gamma_{0} v\right\rangle\right\rangle \rrbracket
$$

so that

$$
\lambda \llbracket\left\langle\left\langle v, f, \gamma_{0} v\right\rangle\right\rangle \rrbracket \leq \llbracket\langle\langle a, b, c\rangle\rangle \rrbracket .
$$

We therefore have

$$
\lambda M^{-1}\|\langle v, f\rangle\| \leq \lambda\left[r\|v\|^{2}+\sigma\|f\|^{2}\right]^{1 / 2} \leq \llbracket\langle\langle a, b, c\rangle\rangle \rrbracket
$$

where $M^{-2}=\min \{r, \sigma\}$. Hence $P(\lambda)$ is bounded and $\|P(\lambda)\| \leq M / \lambda$. If we set $R(\lambda)=$ $B P(\lambda)$ we see that $R(\lambda)\langle\langle a, b, c\rangle\rangle=B\langle v, f\rangle=\left\langle\left\langle v, f, \gamma_{0} v\right\rangle\right\rangle$ and it follows that $R(\lambda)$ is bounded and $\llbracket R(\lambda) \rrbracket \leq 1 / \lambda$. The conditions of Theorem 8.2 are therefore satisfied since 
$Y$ is reflexive. Hence it follows from Theorem 5.2 that the equations $(21),(22)$ under the given boundary conditions are uniquely solvable for any initial state $\langle\langle a, b, c\rangle\rangle \in B[\mathfrak{D}]$.

Acknowledgements. The author is greatly indebted to Christiaan Le Roux and Paul Sauer who carefully read the manuscript and suggested a number of improvements.

\section{References}

[Are87] W. Arendt, Vector-valued Laplace transforms and Cauchy problems, Israel J. Math. 59 (1987), 327-352.

[AF93] W. Arendt and A. Favini, Integrated solutions to implicit differential equations, Rend. Sem. Mat. Univ. Pol. Torino 51 (1993), 315-329.

[CaSh76] R. W. Carroll and R. Showalter, Singular and Degenerate Cauchy Problems, Academic Press, New York, 1976.

[CS94] W. L. Conradie and N. Sauer, Empathy, C-semigroups and integrated semigroups, in: Evolution Equations, Proc. Conf. Baton Rouge 1993, G. Ferreyra, G. R. Goldstein and F. Neubrander (eds.), Lecture Notes in Pure and Appl. Math. 168, Marcel Dekker, New York, 1995, 123-132.

[daP66] G. da Prato, Semigruppi regolarizzabili, Ricerche Mat. 15 (1966), 223-246.

[DP87] E. B. Davies and M. M. Pang, The Cauchy problem and a generalization of the Hille-Yosida theorem, Proc. London Math. Soc. 55 (1987), 181-208.

[DeL91] R. deLaubenfels, Existence and uniqueness families, for the abstract Cauchy problem, J. London Math. Soc. 44 (1991), 310-338.

[DeL94] - Existence Families, Functional Calculus and Evolution Equations, Lecture Notes in Math. 1570, Springer, Berlin, 1994.

[DU77] J. Diestel and J. J. Uhl Jr., Vector Measures, Math. Surveys 15, Amer. Math. Soc., Providence, R.I., 1977.

[Fav79] A. Favini, Laplace transform method for a class of degenerate evolution equations, Rend. Mat. 3-4 (1979), 511-536.

[Fri58] K. O. Friedrichs, Symmetric positive linear differential equations, Comm. Pure Appl. Math. 11 (1958), 333-418.

[HP57] E. Hille and R. S. Phillips, Functional Analysis and Semi-groups, Amer. Math. Soc. Colloq. Publ. 31, 1957.

[Miy51] I. Miyadera, One parameter semi-groups of operators, J. Math. Tokyo 8 (1951), 23-26.

[Sau82] N. Sauer, Linear evolution equations in two Banach spaces, Proc. Roy. Soc. Edinburgh 91A (1982), 387-303.

[Sau95] - Implicit evolution equations and empathy theory, in: Recent Developments in Evolution Equations, Pitman Res. Notes in Math. Ser. 324, A. C. McBride and G. F. Roach (eds.), Longman, Harlow, 1995, 32-39.

[SS87] N. Sauer and J. E. Singleton, Evolution operators related to semigroups of class (A), Semigroup Forum 35 (1987), 317-335.

[SS89] -, -, Evolution operators in empathy with a semigroup, ibid. 39 (1989), 85-94.

[Wid46] D. V. Widder, The Laplace Transform, Princeton Univ. Press, 2nd printing, 1946. 